in: Ensaios Matemáticos | Periodical Volume

1 - 39

\title{
Terms and Conditions
}

The Göttingen State and University Library provides access to digitized documents strictly for noncommercial educational, research and private purposes and makes no warranty with regard to their use for other purposes. Some of our collections are protected by copyright. Publication and/or broadcast in any form (including electronic) requires prior written permission from the Goettingen State- and University Library.

Each copy of any part of this document must contain there Terms and Conditions. With the usage of the library's online system to access or download a digitized document you accept there Terms and Conditions.

Reproductions of material on the web site may not be made for or donated to other repositories, nor may be further reproduced without written permission from the Goettingen State- and University Library For reproduction requests and permissions, please contact us. If citing materials, please give proper attribution of the source.

\section{Contact:}

Niedersächsische Staats- und Universitätsbibliothek

Digitalisierungszentrum

37070 Goettingen

Germany

Email: gdz@www.sub.uni-goettingen.de

\section{Purchase a CD-ROM}

The Goettingen State and University Library offers CD-ROMs containing whole volumes / monographs in PDF for Adobe Acrobat. The PDF-version contains the table of contents as bookmarks, which allows easy navigation in the document. For availability and pricing, please contact:

Niedersaechisische Staats- und Universitaetsbibliothek Goettingen - Digitalisierungszentrum

37070 Goettingen, Germany, Email: gdz@www.sub.uni-goettingen.de 


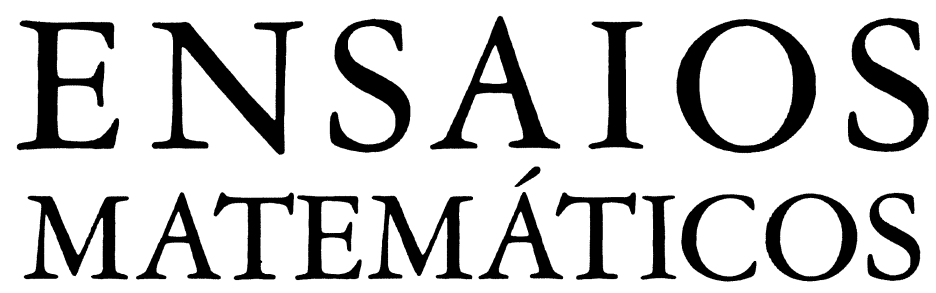

SOCIEDADE BRASILEIRA DE MATEMÁTICA

Équations Differéntielles Linéaires et Transformation de Fourier:

Une Introduction

B. MALGRANGE

Université de Grenoble 


\section{Ensaios Matemáticos}

Esta série objetiva publicar textos sobre temas atuais da Matemática, permitindo ao leitor, através de exposições acessíveis, adquirir ampla perspectiva do assunto em questão, inclusive no que se refere a seus aspectos em aberto. Os trabalhos devem ser enviados a um dos editores.

This series is intended as a vehicle for survey papers in all areas of Mathematics. The aim of the series is to offer readers an accessible overview of current topics in Mathematics. The Brazilian Mathematical Society invites authors to submit papers for inclusion in the series by sending their work to one of the editors.

Full instructions on the preparation of copy are available from the editors upon request.

\section{Editors}

Ju. Il'yashenko

Moscow State University

Department of Mathematics

Moscow - USSR - 117234
R. Moussu

Département de Mathématiques

Université de Dijon

Dijon - France - 21004

$$
\text { P. Sad }
$$

Instituto de Matemática Pura e Aplicada - IMPA

Estrada D. Castorina 110 - J. Botânico

Rio de Janeiro - Brazil - 22460

\section{How to order Ensaios Matemáticos}

Ensaios Matemáticos are available direct from the Brazilian Mathematical Society by writing to the following address:

Ensaios Matemáticos

Sociedade Brasileira de Matemática

Estrada Dona Castorina, 110

Rio de Janeiro 22460

Brazil

๑1989 Sociedade Brasileira de Matemática 
Ces notes reprennent et développent la matière de 3 exposés faits à l'IMPA en mai-juin 88. Leur contenu n'est guère original, et pour l'essentiel il est bien connu des spécialistes. Mais, malgré son caractère élémentaire (au moins, au début), il n'est accessible que dans des articles très spécialisés, et d'un haut niveau de technicité. Peut-être y a-t-il là une lacune, que ces notes aideront à combler. 



\section{B. Malgrange}

\section{$\S 1 \quad$ Introduction}

Considérons un opérateur différentiel linéaire à coefficientes polynomiaux à une variable $p\left(x, \partial_{x}\right)=\sum a_{k, \ell} x^{k} \partial_{x}^{\ell}(x \in \mathbf{C}$; on pose $\partial_{x}=\frac{d}{d x}$ ). Son "transformé de Fourier" $q=\mathcal{F} p$ est l'opérateur en $\xi, \partial_{\xi}$ obtenu par la substitution $\partial_{x} \leftarrow \xi, x \leftarrow-\partial_{\xi}$.

Cherchons à écrire les solutions de $q g=0$ sous la forme $g(\xi)=\int_{\gamma} e^{-x \xi} f(x) d x, f$ et $\gamma$ convenables; en admettant justifiées les dérivations sous le signe $\int$ et les intégrations par parties, on a $(q g)(\xi)=\int_{\gamma}(p f)(x) e^{-x \xi} d x$, et il est naturel de chercher $g$ sous la forme précédente avec $p f=0$. Il est classique ("méthode de Laplace") de faire cela lorsque $q$ est de degré 1 en $\xi$; alors $p$ est de degré 1 en $\partial_{x}$ et l'équation $p f=0$ s'intègre explicitement. En se débrouillant alors pour choisir convenablement $\gamma$, on obtient "en général" - mais pas toujours - toutes les solutions de $q g=0$.

Voici quelques exemples.

Exemple (1.1): On part de l'équation de Bessel

$$
h^{\prime \prime}+\frac{1}{\xi} h^{\prime}+\left(1-\frac{v^{2}}{\xi^{2}}\right) h=0 .
$$

La transformation $h=\xi^{v} g$ nous amène à l'équation

$$
\xi g^{\prime \prime}+(2 v+1) g^{\prime}+\xi g=0
$$

Elle est transformée de Fourier de $\left(1+x^{2}\right) f^{\prime}=(2 v-1) x f$, dont la solution est $f=\left(1+x^{2}\right)^{v-1 / 2}$, à une constante près. 


\section{Équations Différentielles Linéaires}

Pour $v \notin \frac{1}{2}+\mathbf{N}$, on obtient ainsi une base de solutions de $\left(^{*}\right)$ : dans le demi plan $|\arg \xi+\theta|<\frac{\pi}{2}$, on prend $g=\int \gamma_{ \pm i, \theta} f(x) e^{-x \xi} d x$, avec $\gamma_{i, \theta}$ le contour suivant: si $\delta_{i, \theta}$ est la demi-droite d'origine $i$ et d'argument $\theta, \gamma_{i, \theta}$ parcourt $\delta_{i, \theta}$ en partant de l'infini jusqu'au voisinage de $\theta$, contourne $i$ dans le sens direct, et repart sur $\delta_{i, \theta}$ jusqu'à l'infini (cf figure 1).

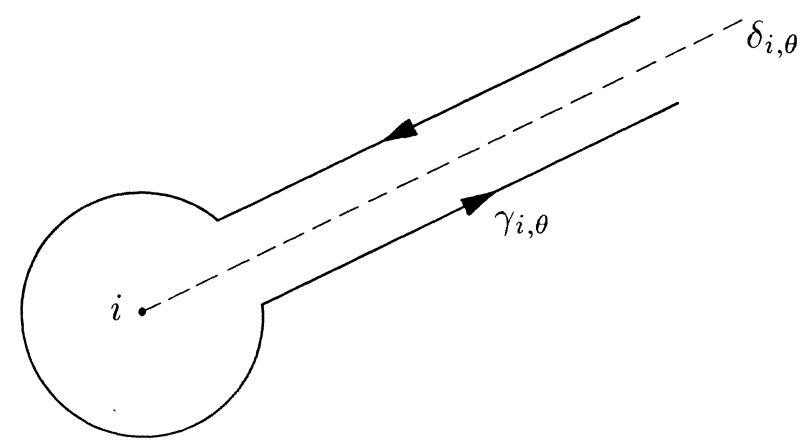

Figure 1

Pour $v=\frac{1}{2}+n, n$ entier $>0$, ceci ne marche pas; notons qu'on peut quand même résoudre l'équation initiale en changeant $v$ en $-v$; mais il reste que la méthode de Laplace ne s'applique pas directement à $\left({ }^{*}\right)$ - Du moins, elle ne semble pas s'y appliquer; nous verrons plus loin comment il faut s'y prendre.

EXemple (1.2): On prend $q$ à coefficients constants, $q=q\left(\partial_{\xi}\right)$; alors $p=p(x) \in \mathbf{C}[x]$, et la méthode ne semble pas s'appliquer, car $p f=0$ entraine $f=0$. Si l'on en croit Ince $[\mathbf{I}]$, c'est Cauchy qui avait vu comment s'en sortir ici: on prend $f=\frac{r}{p}, r$ un polynôme, et $\gamma=$ un cercle entourant tous les zéros de $p$; alors $q g=\int_{\gamma} r e^{-x \xi} d x$; par le théorème de Cauchy, ceci vaut 0 , et on retrouve de cette 


\section{B. Malgrange}

manière les solutions des équations à coefficients constants (qui étaient par ailleurs connues, depuis au moins Euler).

Exemple (1.3): $q g=(\xi g)^{\prime \prime}-g$; alors $p f=x^{2} f^{\prime}-f$ et une base de solutions est $f=e^{-1 / x}$. Une base des solutions de $q g=0$ dans le demi plan $|\arg x+\theta|<\frac{\pi}{2}$ est donnée par $\int_{\gamma_{1}} e^{-1 / x-x \xi} d x$ et $\int_{\gamma_{2}(\theta)} e^{-1 / x-x \xi} d x, \gamma_{1}$ un cercle autour de $0, \gamma_{2}(\theta)$ un chemin partant de 0 dans une direction d'argument $<\frac{\pi}{2}$ en valeur absolue, et allant à l'infini dans la direction $\theta$. (cf figure 2).

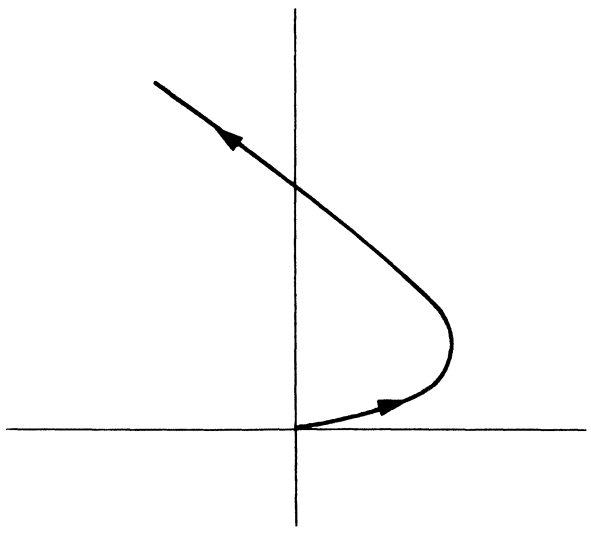

Figure 2

Ce qu'il y a de général derrière ces choix n'est pas bien évident; du reste, je n'éluciderai cette question ici que partiellement. Pour une étude plus complète, voir [Ma 6].

EXEMPLE (1.4): $p f=f^{\prime}+x f$; alors $q g=-g^{\prime}+\xi g$; ici $f=C e^{-x^{2} / 2}$, $g=C^{\prime} e^{\xi^{2} / 2}$; on obtient une représentation de $g$ dans $\mathbf{C}$ tout entier $\operatorname{par} \int_{\mathbf{R}} f(x) e^{-x \xi} d x$, à cause des propriétés de décroissance à l'infini de $f$ dans les directions réelles. 


\section{Équations Différentielles Linéaires}

En fait, je mentionne cet exemple uniquement pour mémoire: le cas où les solutions, ont une croissance d'ordre $>1$ à l'infini est un peu compliqué à étudier en général, et je n'en parlerai pas ici; voir [Ma 6]. Je me limiterai aux équations qui satisfont les conditions de la proposition suivante.

Proposition (1.5). Les propriétés suivantes sont équivalentes:

1) Dans tout secteur $|x|>>0, \alpha<\arg x<\beta$, toutes les solutions de $p f=0$ sont à croissance exponentielle, i.e. vérifient $|f(x)| \leq$ $C e^{A|x|}$, avec $A>0$ et $C>0$ convenables.

2) Soit $m$ (resp. $n$ ) le degré de $p$ par rapport à $x$ (resp. $\partial_{x}$ ). Alors le coefficient de $x^{m} \partial_{x}^{n}$ est $\neq 0$.

3) $q=\mathcal{F} p$ vérifie 1) et 2).

2) $\Rightarrow 1$ ). Hors d'un disque $|x| \leq C$, l'hypothèse $p f=0$ entraine une inégalité $\left|\partial_{x}^{n} f\right| \leq C^{t e} \sum_{p \leq n-1}\left|\partial_{x}^{n} f\right|$; le résultat s'obtient alors par des majorations élémentaires.

La réciproque 1) $\Rightarrow 2$ ) est moins simple, et est un cas particulier de la relation entre la croissance des solutions et le polygone de Newton de l'équation; cette question se décompose en deux: a) La relation entre le polygone de Newton et les solutions formelles. b) La théorie des développements asymptotiques, pour passer des solutions formelles aux solutions effectives. Sur a), voir p.ex. [Ro]; sur b) voir [W]. Sur l'ensemble de la question, voir aussi [Ra 1].

Enfin l'équivalence " $p$ vérifie 2$)$ " $\Leftrightarrow$ " $\mathcal{F} p$ vérifie 2$)$ " est évidente, puisque la condition 2) est symétrique en $x$ et $\partial_{x}$. 


\section{B. Malgrange}

\section{$\S 2$ Microfonctions}

Je reprends ici les notations et la terminologie de [Ma 1], auquel je renvoie pour les détails. Soient $D$ un disque de centre $\mathcal{O} \in \mathbf{C}$, $D^{*}=D-\{0\}$, et $\tilde{D}^{*}$ un revêtement universel de $D^{*}$ (déterminé par le choix d'un point-base $\left.a \in D^{*}\right)$; on note $\mathcal{O}(D)$ et $\mathcal{O}\left(\tilde{D}^{*}\right)$ respectivment l'espace des fonctions holomorphes dans $D$ et $\tilde{D}^{*}$; on pose $\mathcal{C}(D)=\mathcal{O}\left(\tilde{D}^{*}\right) / \mathcal{O}(D)$, et on définit les flèches $\mathcal{O}\left(\tilde{D}^{*}\right) \stackrel{\text { can }}{\rightleftharpoons}$ var $\mathcal{C}(D)$ de la manière suivante (Deligne): "can" est le passage au quotient évident; "var" est l'unique application telle qu'on ait (var)o(can) $=T-\mathrm{id}, T$ l'action de la monodromie (= d'un lacet à partir de $a$ dans le sens direct) sur $\mathcal{O}\left(\tilde{D}^{*}\right)$.

Si l'on fait tendre le rayon de $D$ vers 0 (on déplace p.ex. le point-base radialement), on obtient des espaces notés respectivement $\mathcal{O}, \tilde{\mathcal{O}}, \mathcal{C}$, et des flèches $\tilde{\mathcal{O}} \stackrel{\text { can }}{\rightleftharpoons}$ var $\mathcal{C}$.

L'espace $\mathcal{C}$ est une variante des espaces introduits par Sato (voir notamment [SSK]) sous les noms d'hyperfonctions et de microfonctions. C'est pourquoi j'appelerai ses éléments "microfonctions". Ses propriétés sont très voisines de celles de l'espace des germes en $0 \in \mathbf{R}$ de distributions (ou d'hyperfonctions) à support dans $\mathbf{R}_{+}$:

a) On ne peut pas multiplier deux éléments de $\mathcal{C}$, mais un élément de $\mathcal{O}$ et un de $\mathcal{C}$.

b) Par contre, la convolution est définie sur $\mathcal{C}$, et en fait une algèbre commutative et associative. L'unité est la "(micro)fonction de Dirac" $\delta=\operatorname{can}\left(\frac{1}{2 \pi i x}\right)$.

c) L'action de $\partial_{x}$ sur $\mathcal{C}$ est bijective (deux primitives diffèrent par une constante, qui s'annule par le passage au quotient). Comme on a $\partial_{x}(f * g)=\left(\partial_{x} f\right) * g=f *\left(\partial_{x} g\right)$, l'intégration est 


\section{Équations Différentielles Linéaires}

égale à la convolution par la primitive de $\delta$; celle-ci, notée $Y$ (microfonction de Heaviside) est égale à can $\left(\frac{1}{2 \pi i} \log x\right)$.

Notons aussi que la bijectivité de $\partial_{x}$ permet de définir l'action sur $\mathcal{C}$ d'opérateurs plus généraux que les opérateurs différentiels linéaires, à savoir les opérateurs pseudo-différentiels de Boutet de Monvel-Krée [B-K]. Ce fait, et son extension à plusieurs variables, sont le point de départ de l'analyse microlocale à la Sato [SKK].

THÉORÈmE (2.1). (Kashiwara). Soit $p=\sum_{0}^{n} a_{k} \partial_{x}^{k}$ un opérateur différentiel linéaire, à coefficients dans $\mathcal{O}$, avec $a_{n} \neq 0$. Notons $m=v\left(a_{n}\right)$ l'ordre du zéro de $a_{n}$ en 0 . Alors, l'application $p: \mathcal{C} \rightarrow \mathcal{C}$ est surjective, et son noyau est de dimension $m$.

Démonstration: Considérons la suite exacte $0 \rightarrow \mathcal{O} \rightarrow \tilde{\mathcal{O}} \rightarrow \mathcal{C} \rightarrow$ $O$; en lui appliquant $p$, on trouve une suite exacte à 6 termes

$$
\begin{aligned}
0 \rightarrow \operatorname{ker}(p, \mathcal{O}) & \rightarrow \operatorname{ker}(p, \tilde{\mathcal{O}}) \rightarrow \operatorname{ker}(p, \mathcal{C}) \\
& \rightarrow \operatorname{coker}(p, \mathcal{O}) \rightarrow \operatorname{coker}(p, \tilde{\mathcal{O}}) \rightarrow \operatorname{coker}(p, \tilde{\mathcal{C}}) \rightarrow 0
\end{aligned}
$$

Le théorème d'existence et d'unicité pour les équations différentielles nous dit que $\operatorname{ker}(p, \tilde{\mathcal{O}})$ est de dimension $n$, et qu'on a $\operatorname{coker}(p, \tilde{\mathcal{O}})=0$; donc on aura aussi $\operatorname{coker}(p, \mathcal{C})=0$, d'òu la première assertion.

Pour démontrer la seconde, il suffit alors d'appliquer le théorème de l'indice (dû indépendamment à Kashiwara, Komatsu, et l'auteur, voir p.ex. [Ma 1]); il dit ceci: le noyau et le conoyau de $p$ appliqué à $\mathcal{O}$ sont de dimension finie, et la différence de ces dimensions est $n-m$. En utilisant cela, et le fait que la somme alternée des dimensions des espaces figurant dans (2.2) est nulle, on obtient le résultat cherché. 


\section{B. Malgrange}

(A noter que Kashiwara procède en sens inverse: il prouve d'abord (2.1) par analyse microlocale et en déduit le théorème de l'indice (voir $[\mathbf{K}])$.)

Soit maintenant $f \in \mathcal{C}$. Supposons que, le long de la demi droite $\delta_{0, \theta}$ d'origine 0 et d'argument $\theta$, var $f$ se prolonge en une fonction holomorphe $g$, à croissance exponentielle à l'infini; on appellera "hyperfonction (dans la direction $\delta_{0, \theta}$ )" et on notera $F=(f, g)$ cette paire de données. On définit la transformée de Fourier (ou FourierLaplace) de $F$ de la manière suivante: soit $\tilde{f} \in \tilde{\mathcal{O}}$, avec can $\tilde{f}=f$. Soient $b \in \delta_{0, \theta}$, assez voisin de $0, \gamma_{b}^{\prime}$ l'intervalle $\left[b, \infty\left[\right.\right.$ de $\delta_{0, \theta}$, parcouru de 0 à l'infini, et $\gamma_{b}^{\prime \prime}$ le cercle de centre 0 et de rayon $|b|$, parcouru dans le sens direct à partir de $b$. Alors, on pose

$$
\bar{\int}_{\delta_{0, \theta}} F e^{-x \xi} d x=\int_{\gamma_{b}^{\prime}} g(x) e^{-x \xi} d x+\int_{\gamma_{b}^{\prime \prime}} \tilde{f}(x) e^{-x \xi} d x
$$

cette intégrale ne dépend pas de $b$ (parce que $T \tilde{f}-\tilde{f}=g$ ), et elle ne dépend pas non plus du représentant $\tilde{f}$ choisi. Comme $g$ est à croissance exponentielle elle converge pour $\xi$ vérifiant $\operatorname{Re}\left(\xi e^{i \theta}\right)>>0$. Il est clair aussi que, si l'on a une microfonction définie (de la manière évidente) au voisinage d'un autre point $a \in \mathbf{C}$, et possédant une propriété analogue de prolongement analytique le long d'une demidroite $\delta_{a, \theta}$, on pourra de la même manière définir la transformée de Laplace de l'hyperfonction ainsi définie; ce sera une fonction holomorphe dans un demi plan $\operatorname{Re}\left(\xi e^{i \theta}\right)>>0$, et, à l'infini, elle sera à croissance exponentielle dans tout secteur $|\arg \xi+\theta| \leq \frac{\pi}{2}-\varepsilon, \varepsilon>0$.

Soit en particulier $p=\sum_{0}^{n} a_{k} \partial_{x}^{k}$, avec $a_{i} \in \mathbf{C}[x]$, un opérateur différentiel vérifiant les conditions (1.5), et soient $\alpha_{1}, \ldots, \alpha_{r}$ ses points singuliers, i.e. les points où $a_{n}$ s'annule; si $m_{k}$ est la multiplicité de $\alpha_{k}$, on a $\sum m_{k}=m\left(=d^{o} a_{n}\right)$. Soit $\theta$ une direction 


\section{Équations Différentielles Linéaires}

telle que les demi-droites $\delta_{\alpha_{k}, \theta}$ ne se rencontrent pas. D'après le théorème précédent, on peut fabriquer $m_{k}$ microfonctions $f$ en $a_{k}$, linéairement independantes, solutions de $p f=0$, et elles se prolongent le long de $\delta_{\alpha_{k}, \theta}$ en des hyperfonctions solutions $F$ qui sont à croissance exponentielle. On a alors le resultat suivant

ThÉORÈme (2.2). Dans un demi-plan $\operatorname{Re}\left(\xi e^{i \theta}\right)>>0$, les transformés de Fourier des hyperfonctions solution de $p F=0$ définies le long des lignes $\delta_{\alpha_{k}, \theta}$ par le procédé précédent forment une base des solutions de l'équation $q g=0(q=\overline{\mathcal{F}} p)$.

A remarquer qu'a priori, on a "le compte" de solutions, car $\sum m_{i}=m=\operatorname{deg} q / \partial_{\xi} ;$ mais il faut montrer leur indépendance; la démonstration de ce théorème sera donnée au paragraphe 4. Auparavant, reprenons les exemples du $\S 1$.

EXEMPLE (1.1): (Une détermination convenable de) $\left(1+x^{2}\right)^{v-1 / 2}$, prise au voisinage de $\pm i$, donne, par "can", les microfonctions solutions de l'équation $\left(1+x^{2}\right) f^{\prime}-(2 v-1) x f=0$; ceci lorsque $v \notin \frac{1}{2}+\mathrm{N}$.

Pour $v=\frac{1}{2}+n$, ceci ne marche pas, et il faut déterminer directement les microfonctions solutions; par exemple, pour $v=\frac{1}{2}$, on a à résoudre l'équation $\left(1+x^{2}\right) f^{\prime}=0$; les microfonctions solutions en $\pm i$ sont $Y_{ \pm i}$ (les translatées de la microfonction d'Heaviside). Les solutions de $\left(^{*}\right)$ sont donc données par

$$
g_{ \pm}(\xi)=\int_{\gamma \pm i, \theta} e^{-x \xi} d x=\frac{e^{\mp i \xi}}{\xi}
$$

la vérification directe de ce resultat est immédiate. Je laisse le lecteur regarder de même les cas $v=\frac{1}{2}+n, n \geq 1$. 


\section{B. Malgrange}

EXemple (1.2): Ici on doit chercher les solutions de $p(x) f=$ 0 . Si $\alpha_{1}, \ldots, \alpha_{\ell}$ sont les zéros de $p$, de multiplicités respectives $m_{1}, \ldots, m_{\ell}$, les microfonctions solutions sont les $\partial_{x}^{k} \delta_{\alpha_{i}}$ avec $k \leq m_{i}$, $\delta_{\alpha_{i}}$ la microfonction de Dirac en $\alpha_{i}$, et l'on retrouve immédiatement les formules usuelles.

EXEMPLE (1.3): Une base de microfonctions solutions de $x^{2} f^{\prime}-$ $f=0$ est obtenue ainsi

i) on prend $f_{1}=\operatorname{can} e^{-1 / x}$

ii) on prend la fonction $\mathcal{C}^{\infty}$ sur $\mathbf{R}$ (au voisinage de 0 ) qui vaut $e^{-1 / x}$ pour $x>0$ et 0 pour $x \leq 0$; on l'écrit (par l'intégrale de Cauchy) comme différence des valeurs au bord de 2 fonctions holomorphes, $f_{+}$et $f_{-}$, respectivement dans $\operatorname{Im} x>0$ et $\operatorname{Im} x<0$; donc $f_{+}$et $f_{-}$seront le prolongement analytique l'une de l'autre pour $x<0$; et, pour $x>0$, on aura $f_{-}-f_{+}=e^{-1 / x} ;$ il est immédiat que la fonction holomorphe ainsi obtenue dans $\mathbf{C}-\mathbf{R}_{+}$(au voisinage de 0 ) se prolonge en un élément $\tilde{f}$ de $\tilde{\mathcal{O}}$, on pose $f_{2}=\operatorname{can} \tilde{f}$; à remarquer qu'ici, on a var $f_{2}=e^{-1 / x}$.

iii) Une autre méthode est la suivante: on prend $g \in \mathcal{O}$, tel que l'équation $x^{2} \tilde{f}^{\prime}-\tilde{f}=g$ n'ait pas de solution dans $\mathcal{O}$, on prend une solution $\tilde{f}$ de cette équation dans $\tilde{\mathcal{O}}$, et on prend $f=\operatorname{can} \tilde{f}$. EXERCICE: prendre $g=-x$, et prendre la solution $\tilde{f}$ qui admet pour développement asymptotique dans $\operatorname{Re} x<0$ la série d'Euler $\sum n ! x^{n+1}$. Exprimer can $\tilde{f}$ en fonction de $f_{1}$ et $f_{2}$. 


\section{Équations Différentielles Linéaires}

\section{§3 Transformation de Fourier dans le domaine complexe}

Pour démontrer et généraliser le Théorème (2.2), j'aurai besoin de quelques résultats sur la transformation de Fourier dans le domaine complexe; les résultats que je donnerai ici sont élémentaires, et très partiels. Pour des résultats plus systematiques, voir [Ma 5] et sa bibliographie.

Il sera commode ici d'utiliser le langage suivant: soit $\overline{\mathbf{C}}$ le compactifié de C (côté "variable $\xi$ ") par le cercle $S$ des directions de demi-droites; pour $\tau \in S$, on note $\mathcal{V}(\tau)$ l'ensemble des ouverts $U \subset \mathbf{C}$ dont l'adhérence dans $\overline{\mathbf{C}}$ est un voisinage de $\tau$ (autrement dit, $U$ contient un ensemble de la forme $\left.|\xi|>\frac{1}{\varepsilon},|\arg \xi-t|<\varepsilon\right)$. Soit $\mathcal{A}^{<1}$ le faisceau sur $S$ ainsi défini: en $\tau \in S, \mathcal{A}_{\tau}^{<1}$ est l'espace des $g$ holomorphes dans un $U \in \mathcal{V}(\tau)$ et qui y sont à croissance sous-exponentielle, c'est-à-dire vérifient la condition suivante:

$$
\forall \varepsilon>0, \exists c>0 \text { avec } \forall \xi \in U,|g(\xi)| \leq C e^{\varepsilon|\xi|} .
$$

On prend ensuite les germes en $\tau$, i.e. on passe à la limite inductive suivant $\mathcal{V}(\tau)$.

Considérons d'autre part un ensemble d'hyperfonctions un peu plus général que celui du $§ 2$, défini ainsi: une hyperfonction $F$ sur $\delta_{0, \theta}$ est définie par la donnée:

i) d'une fonction holomorphe $f$ à croissance exponentielle à l'infini dans un secteur $|\arg x-\theta|<\varepsilon$

ii) d'une fonction $\tilde{f}$ holomorphe sur un secteur ramifié $\{|x|<$ $\varepsilon ;-\varepsilon<\arg x<2 \pi+\varepsilon\}$ vérifiant là où on pense $\tilde{f}\left(e^{2 \pi i} x\right)-$ $\tilde{f}(x)=f(x)$. 


\section{B. Malgrange}

Deux telles paires $(f, \tilde{f})$ et $\left(f_{1}, \tilde{f}_{1}\right)$ définissent la même hyperfonction $F$ si $f=f_{1}$ et $\tilde{f}=\tilde{f}_{1}$ est holomorphe en 0 (en outre, on passe à la limite inductive pour $\varepsilon \rightarrow 0$ ).

Comme au $\S 2$, on définit le transformé de Fourier (Laplace) $G=\mathcal{F F}$; c'est une fonction holomorphe et à croissance sousexponentielle à l'infini, définie dans un ouvert $U$ formé des $\xi$ qui vérifient $\operatorname{Re}\left(e^{i \theta^{\prime}} \xi\right)>C$ pour un $\theta^{\prime}$ voisin de $\theta$; autrement dit, en posant $I_{\theta}=\left\{\tau ;|\tau+\theta| \leq \frac{\pi}{2}\right\}$, c'est une section de $\mathcal{A}^{<1}$ sur $I_{\theta}$ (ou sur un voisiange de $I_{\theta}$, c'est pareil par définition).

Proposition (3.2). La transformation de Fourier est une bijection de l'espace des hyperfonctions sur $\delta_{0, \theta}$ (au sens qui vient d'être défini) sur l'espace $\Gamma\left(I_{\theta}, \mathcal{A}^{<1}\right)$.

L'application inverse se définit ainsi: soit $G$ défini dans un ouvert $U$ et à croissance sous-exponentielle comme ci-dessus; on prend $a \in U$ et on considère l'intégrale $\frac{-1}{2 \pi i} \int_{\delta_{a, r}} G(\xi) e^{x \xi} d \xi$ lorsque $\tau$ parcourt un intervalle $|\tau+\theta|<\frac{\pi}{2}+\varepsilon$; on obtient ainsi une fonction holomorphe sur le secteur ramifié $\theta-\varepsilon<\arg x<\theta+2 \pi+\varepsilon$ qui définit de façon évidente une paire $(\tilde{f}, f)$. Pour montrer qu'elle donne l'inverse cherchée, on permute les intégrations et on applique la formule de Cauchy, ceci un certain nombre de fois. Les détails sont un peu longs, mais sans difficulté sérieuse, et je les laisse au lecteur. Voir des calculs très voisins dans [B].

VARIANTE (3.3): Soit $\mathcal{A}^{\leq-1}$ (resp. $\mathcal{A}_{\bar{B}}^{\leq-1}$, pour $B>0$ ) le faisceau sur $S$ des fonctions holomorphes à décroissance exponentielle (resp. de type $B$ ), c'est-à-dire telles qu'il existe $A>0, C>0$ tels qu'on ait $|g(\xi)| \leq C e^{-A|\xi|}$ (resp. il existe $C>0$ tel qu'on ait $|g(\xi)| \leq$ $\left.C e^{-B|\xi|}\right)$.

Définissons d'autre part, comme nous l'avons fait pour les 


\section{Équations Différentielles Linéaires}

hyperfonctions, des microfonctions un peu plus générales que l'espace $\mathcal{C}$ : une "microfonction dans la direction $\theta$ " est un $\tilde{f}$ holomorphe dans $0<|x|<\varepsilon,-\varepsilon<\arg x<2 \pi+\varepsilon$, modulo les $\tilde{f}$ holomorphes en 0 et le passage au limite inductive $\varepsilon \rightarrow 0$. Leur ensemble sera noté $\mathcal{C}_{\theta}$; alors on a une bijection $\mathcal{F}: \mathcal{C}_{\theta} \stackrel{\sim}{\longrightarrow}$ sections sur $I_{\theta}$ de $\mathcal{A}^{<1} / \mathcal{A}^{\leq-1}$ (l'application directe se définit comme pour les hyperfonctions, mais en ne gardant que la partie du contour d'intégration située près de 0 , l'application inverse se définit de manière analogue à ci-dessus).

En particulier, la transformation de Fourier donne une application de $\mathcal{C}$ dans l'espace des sections de $\mathcal{A}^{<1} / \mathcal{A}^{\leq-1}$ sur $\tilde{S}$, revêtement universel de $S$; mais la réciproque n'est pas vraie (de telles sections donnent des microfonctions $\theta \mapsto \mathcal{C}_{\theta}$ dont le domaine d'existence peut tendre vers 0 lorsqu'on fait tourner $\theta$ ). La correspondance exacte est la suivante $\mathcal{F C} \simeq U_{B}$ (sections sur $\tilde{S}$ de $\mathcal{A}^{<1} / \mathcal{A}_{\bar{B}}^{\leq-1}$ ).

Remarque (3.4): Pour $\tau \in S$, l'espace $\mathcal{C}_{\tau}^{\mathbf{R}}$ des "microfonctions de Sato $[\mathbf{S K K}],[\mathbf{K}]$ dans la codirection $\tau$ " est une fonction $\tilde{f}$ holomorphe dans $|x|<\varepsilon, \frac{\pi}{2}-\varepsilon<\arg x+\tau<\frac{3 \pi}{2}+\varepsilon$, modulo les $\tilde{f}$ holomorphes en 0 , et le passage à la limite $\varepsilon \rightarrow 0$. On voit aussi qu'on a $\mathcal{F C}_{\tau}^{\mathbf{R}}=\mathcal{A}_{\tau}^{<1} / \mathcal{A}_{\bar{\tau}}^{\leq-1}$, et que $\mathcal{C}_{\theta}$ est l'espace des sections de $\mathcal{C}_{\tau}^{\mathbf{R}}$ sur l'intervalle $I_{\theta}$. Je n'utiliserai pas ces résúltats dans la suite.

Cette remarque est liée à la suivante: malgré les apparences, et si l'on fait abstraction des conditions de croissance, les constructions du type précédent sont essentiellement symétriques par rapport aux variables $x$ et $\xi$. Cela résulte des formules "d'inversion géométrique" dans la transformation de Fourier. Voir à ce sujet [SKK] et la bibliographie de [Ma 5]. 


\section{B. Malgrange}

\section{§4 Démonstration du Théorème (2.2), et com- pléments}

(4.1) Reprenons les notations de (2.2); soit $F_{\alpha_{k}, j}$ une base des hyperfonctions solutions de $p F=0$, prises le long des $\delta_{\alpha_{k}, \theta}(1 \leq j \leq$ $m_{k}$ ); en fait, elles sont définies et à croissance exponentielle dans un petit secteur autour de $\delta_{\alpha_{k}, \theta}$; si l'on note $G_{\alpha_{i}, j}$ les transformées de Fourier, on aura donc, d'après (3.2) $e^{\alpha_{k} \xi} G_{\alpha_{k}, j} \in \Gamma\left(I_{\theta}, \mathcal{A}^{<1}\right)$. Pour $k$ fixé, les $F_{\alpha_{k}, j}$ sont linéairement indépendantes; donc d'après (3.2), leurs transformées de Fourier le seront aussi. Reste à démontrer qu'il n'y a pas de relation $\sum H_{k}=0, H_{k}$ étant une combinaison $\operatorname{des} G_{\alpha_{k}, j}$. On a $H_{k}=e^{-\alpha_{k} \xi} H_{k}^{\prime}$, avec $H_{k}^{\prime} \in \Gamma\left(I_{\theta}, \mathcal{A}^{<1}\right)$.

Supposons l'existence d'une telle relation, et montrons p.ex. qu'on a $H_{1}=0$. Il existe une direction $\tau \in I_{\theta}$ telle qu'on ait $\operatorname{Re}\left(\alpha_{1} e^{i r}\right)<\operatorname{Re}\left(\alpha_{k} e^{i r}\right), k \geq 2$; la même inégalité sera encore vraie pour $\tau^{\prime}$ voisin de $\tau$; il résulte de là que, dans un secteur $U \in \mathcal{V}(\tau)$, $H_{1}^{\prime}$ sera à décroissance exponentielle; choisissons alors $a$ tel que $\delta_{a, r} \subset U$; la fonction $\int_{\alpha, \tau} H_{1}^{\prime}(\xi) e^{x \xi} d \xi$ est holomorphe au voisinage de 0 ; le procédé d'inversion de la transformation de Fourier indiqué en (3.2) nous montre alors que la microfonction correspondante est nulle. On en déduit $H_{1}^{\prime}=0$, et $H_{1}=0$, d'où le théorème.

(4.2) Considérons, de manière analogue à ce qu'on a fait au $§ 3$, les solutions de l'équation $q g=0$ au voisinage de l'infini comme un faisceau sur $S$, faisceau qu'on notera $E$. Les résultats précedents nous donnent en prime l'énoncé suivant:

(4.2.1). Sur un demi-cercle $I \subset S$, dont les extrémités "ne sont pas des lignes de Stokes", i.e. vérifient $\operatorname{Re}\left(\alpha_{j}-\alpha_{k}\right) e^{i r} \neq 0, \forall j, k$, 


\section{Équations Différentielles Linéaires}

on a une décomposition unique de $\Gamma(I, E)=E(I)$ en sous-espaces $E_{\alpha_{j}}(I)$ tels que $g$ appartient à $E_{\alpha_{j}}(I)$ ssi l'on a $e^{\alpha_{j} \xi} g \in \Gamma\left(I, \mathcal{A}^{<1}\right)$ (autrement dit si $e^{\alpha_{j} \xi} g$ est à croissance sous-exponentielle sur I).

Introduisons, pour tout $\tau \in S$, l'ordre partiel suivant sur l'ensemble $\left\{\alpha_{j}\right\}: \alpha_{j} \leq_{\tau} \alpha_{k}$ si, pour $\tau^{\prime}$ voisin de $\tau$, on a $\operatorname{Re}\left(\alpha_{j}-\right.$ $\left.\alpha_{k}\right) e^{i \tau^{\prime}} \geq 0$ (ceci équivaut à dire qu'on a, soit $\alpha_{j}=\alpha_{k}$, soit $\left.\operatorname{Re}\left(\alpha_{j}-\alpha_{k}\right) e^{i r}>0\right)$. Soit, pour $\tau \in S, E_{\tau}^{\alpha_{j}}$ le sous-espace des $g \in E_{\tau}$ qui, dans un $U \in \mathcal{V}(\tau)$ sont tels que $E^{\alpha_{j} \xi} g$ soit à croissance sous-exponentielle. Le résultat précédent implique en particulier ceci:

(4.2.2). En tout point $\tau \in S$, il existe une décomposition (non unique en général) $E_{\tau}=\oplus E_{\alpha_{j}}$ telle que, pour $\tau^{\prime}$ voisin de $\tau$, on ait $E_{\tau^{\prime}}^{\alpha_{j}}=\bigoplus_{\alpha_{k} \leq \tau^{\prime} \alpha_{j}} E_{\alpha_{k}}$ (on identifie $E_{\tau}$ à $E_{\tau^{\prime}}$ en profitant de ce que $E$ est un système local).

Le système local $E$, muni de la filtration $E^{\alpha_{j}}$ satisfaisant (4.2.2) est un cas particulier de ce qu'on appelle une "structure de Stokes" (pour la commodité, je le qualifierai dans la suite de cet exposé du nom de "structure de Stokes de niveau 1"). Pour la notion générale de structure de Strokes, et son rôle dans la classification des points singuliers des équations différentielles linéaires, voir [Ma 3].

Remarquons aussi que (4.2.1) semble a priori plus fort que (4.2.2), mais en fait on peut voir (même reférence, par exemple) que (4.2.2) entraine (4.2.1).

(4.2.3) Il est enfin intéressant d'examiner comment se fait le changement de décomposition ("phénomène de Stokes") lorsqu'on passe d'un intervalle fermé $I$ de longueur $\pi$ à un autre $I^{\prime}$ tel que $I-I^{\prime}$ contienne une ligne de Stokes, et donc $I^{\prime}-I$ la ligne de Stokes 


\section{B. Malgrange}

"opposée". En particulier, il est intéressant de voir comment cela se lit "du côté des $x$ " i.e. du côté hyperfonctions. Le cas le plus simple est celui où une demi-droite $\delta_{\alpha_{j}, \theta}$ passe par un unique $\alpha_{k} \neq \alpha_{j}$, disons pour $\theta=\theta_{o}$; soient $\theta_{-}$et $\theta_{+}$deux valeurs voisines de $\theta_{o}$, qui lui soient respectivement inférieure et supérieure. Il est immédiat de voir que les choses se passent ainsi: $E_{\alpha_{j}}(I) \oplus E_{\alpha_{k}}(I)$ ne change pas lorsqu'on passe de $I=I_{\theta_{-}}$à $I=I_{\theta_{+}}$; de plus, dans ces passages les éléments du sous-espace $E_{\alpha_{k}}(I)$ sont fixes individuellement, ainsi que ceux du quotient (j'identifie ici, bien sur, $E\left(I_{\theta_{-}}\right)$à $E\left(I_{\theta_{+}}\right)$. en utilisant le fait que $E$ est un système local). Donc le passage se traduit par une application $E_{\alpha_{j}}\left(\theta_{-}\right) \rightarrow E_{\alpha_{k}}\left(\theta_{-}\right)=E_{\alpha_{k}}\left(\theta_{o}\right)$ qui se traduit ainsi du coté microfonctions, modulo les conventions convenables sur les points-base et les déterminations: on prend une microfonction solution de $p f=0$ au voisinage de $\alpha_{j}$; on prolonge analytiquement var $f$ le long de segment $] \alpha_{j}, \alpha_{k}$ [ et on prend le "can" changé de signe de la fonction ainsi obtenue au voisinage de $\alpha_{k}$. Je laisse le lecteur écrire les choses en détails et généraliser au cas òu $\delta_{\alpha_{j}, \theta_{o}}$ contient plusieurs points $\alpha_{k}$; tout ceci ne contient essentiellement rien d'autre que les "déformations de contour" de l'analyse classique lorsqu'on passe un point singulier.

(4.3) La théorie des structures de Stokes s'applique généralement aux systèmes méromorphes différentiels au voisinage d'un point singulier. En particulier, la théorie des structures de niveau 1, i.e. les propriétés (4.2.1) et (4.2.2) seront vérifiées sans changement par les solutions d'un système $\partial_{\xi} G+M G=0$, avec $M$ une matrice carrée $n \times n$ holomorphe à l'infini: $M=M_{o}+\frac{M_{1}}{\xi}+\frac{M_{2}}{\xi^{2}}+\ldots$ (c'est la condition qui assure la croissance exponentielle; le lecteur pourra vérifier que ceci contient les équations satisfaisant (1.5) comme cas 


\section{Équations Différentielles Linéaires}

particulier). Ici les $\alpha_{k}$ sont les valeurs propres de $M_{o}$, et les $m_{k}$ leurs multiplicités dans le polynôme caractéristique de $M_{o}$. Ces résultats s'établissent par les procédés habituels dans le sujet;

a) forme normale formelle, en particulier décomposition formelle suivant les valeurs propres de $M_{o}$ ("lemme de Sibuya")

b) théorie des développements asymptotiques, qui dit que la mise sous forme normale formelle se "réalise analytiquement" dans tout secteur d'ouverture assez petite. Voir p.ex. sur ces points $[\mathbf{L}]$ et $[\mathbf{W}]$.

On peut se demander si, ici aussi, ces résultats peuvent se récupérer par transformation de Fourier. La réponse est oui, et on a le résultat suivant.

ThÉorÈme (4.3.1). (Ecalle) Soient $\alpha_{1}, \ldots, \alpha_{\ell}$ les valeurs propres distinctes de $M_{o}$, et soient $m_{1}, \ldots, m_{\ell}$ leurs multiplicités (dans le polynôme caractéristique de $M_{o}$ ). Pour $k=1, \ldots, \ell$, on peut trouver $m_{k}$ microfonctions en $\alpha_{k}$ (i.e. éléments de $\mathcal{C}$, translatés par $\alpha_{k}$ ) $f_{\alpha_{k}, j}$, linéairement indépendantes, et qui possédent les propriétés suivantes

i) Leurs images par "var" se prolongent en des fonctions holomorphes sur le revêtement universel de $\mathbf{C}-\left\{\alpha_{1}, \ldots, \alpha_{\ell}\right\}$; de plus, dans tout secteur à l'infini ne contenant pas de singularité, ce prolongement est à croissance exponentielle

ii) Si $\theta$ est une direction de $1 / 2$ droites telle que les $\delta_{\alpha_{k}, j}$ ne se recontrent pas, désignons par $F_{\alpha_{k}, j}$ les hyperfonctions définies par les prolongements des $f_{\alpha_{k}, j}$ le long de $\delta_{\alpha_{k}, j}$. Alors les $\mathcal{F} F_{\alpha_{k}, j}$ forment une base des solutions de $\partial_{\xi}+M G=0$ sur $I_{\theta}$.

Ce résultat est en fait un cas particulier des résultats démontrés 


\section{B. Malgrange}

par Ecalle pour des équations différentilles linéaires ou non, et pour d'autres "objets locaux". La méthode de cet auteur consiste essentiellement à transformer, par Fourier, l'équation donnée en une équation intégrale de convolution, équation que l'on résoud par des procédés d'itération; voir $[\mathbf{E}]$.

On peut se demander si l'on peut aussi procéder en sens inverse, i.e. déduire (4.3.1) de la théorie des structures de Stokes, via transformation de Fourier. La réponse est très probablement positive (les microfonctions se fabriquent facilement; le point à vérifier est l'existence du prolongement analytique, et la majoration à l'infini; une fois ceci obtenu la manière dont se fait le prolongement analytique résulte du dictionnaire esquissé en (4.2.3)). Toutefois, comme je n'ai pas regardé la question en détail, je ne me prononcerai pas de manière catégorique.

\section{$\S 5$ Microfonctions régulières}

(5.1) Je reprend ici les notations du §2. Suivant une terminologie maintenant bien établies, on dit qu'un $f \in \tilde{\mathcal{O}}$ est de détermination finie si l'espace vectoriel qu'elle engendre sous l'action de la monodromie est de dimension finie. Il est classique que ceci équivaut à la condition suivante: $f=\sum f_{\alpha, p} x^{\alpha}(\log x)^{p}(\alpha \in \mathbf{C}, p \in \mathbf{N}$; somme finie), avec $f_{\alpha, p}$ holomorphes sur un disque pointé $D^{*}$ [Indication sur la démonstration: prendre une base $\Phi=\left(f_{1}, \ldots, f_{p}\right)$ de cet espace tel que $T \Phi=\Phi C, C \in G \ell(n, \mathbf{C})$ étant sous forme de Jordan; prendre un $\Gamma$ tel qu'on ait $\exp (2 \pi i \Gamma)=C$ et considérer $\Phi \cdot \exp (-\Gamma \log x)]$. 


\section{Équations Différentielles Linéaires}

On dit que $f \in \tilde{\mathcal{O}}$ est "de classe de Nilsson" si elle est de détermination finie et si, dans tout secteur $\alpha<\arg x<\beta, f$ est à croissance modérée (i.e. plus petite qu'une puissance de $\frac{1}{|x|}$ ) près de 0 . $\mathrm{Si}$, dans l'expression ci-dessus de $f$, on suppose les $\alpha$ distincts modulo $\mathbf{Z}$, cette dernière condition équivaut au fait que les $f_{\alpha, p}$ sont méromorphes en 0 ; on peut même, si l'on veut, en décalant les $\alpha$, les supposer holomorphes.

On dira aussi qu'une microfonction $g \in \mathcal{C}$ est de détermination finie (resp. de classe de Nilsson) si $g=\operatorname{can} f, f \in \tilde{\mathcal{O}}$ de détermination finie (resp. de classe de Nilsson).

Si $p=\sum a_{k} \partial_{x}^{k}\left(a_{k} \in \mathcal{O}\right)$ est un opérateur différentiel linéaire $\neq 0$, ses solutions dans $\tilde{\mathcal{O}}$ sont toujours de détermination finie; elles sont toutes de classe de Nilsson si et seulement si $p$ est à singularité régulière (théorie de Fuchs; voir p.ex. [I]); d'autre part, il est facile de voir que toute fonction de classe de Nilsson est solution d'une équation à singularité régulière. Je laisse au lecteur le soin d'énoncer et de démontrer les propriétés analogues pour les microfonctions de classe de Nilsson.

(5.2) Dans la fin de ces notes, et uniquement dans un but de simplification des notations, je me limiterai à l'étude de celles des microfonctions de classe de Nilsson qui sont invariantes par la monodromie; il est facile de voir que ce sont celles qui s'écrivent

$$
f=\varphi Y+\sum_{0 \leq k \leq m} a_{k} \delta^{(k)},
$$

$\delta^{(k)}$ la dérivée d'ordre $k$ de $\delta, \varphi \in \mathcal{O}$; écrivant $\delta^{(-k)}$ pour la primitive d'ordre $k$ de $\delta$, on a $\delta^{(-k)}=\frac{x^{k-1}}{(k-1) !} Y$, d'où

$$
f=\sum_{k \leq m} a_{k} \delta^{(k)}
$$




\section{B. Malgrange}

avec $\varphi(x)=\sum_{k \geq 1} a_{-k} \frac{x^{k-1}}{(k-1) !}$

Je qualifierai ces microfonctions de "régulières", et noterai $\mathcal{C}_{o}$ leur ensemble; mais il est entendu que tout ce qui va être dit s'étend sans modification (sauf dans les complication des formules) aux microfonctions de classe de Nilsson.

(5.3) Prenons $f \in \mathcal{C}_{o}$, comme ci-dessus et soit $g \in \Gamma\left(S, \mathcal{A}^{<1} / \mathcal{A}^{\leq-1}\right)$ sa transformée de Fourier au sens de (3.3); avec les notations du §3, sur l'intervalle $I_{\theta}$ un représentant de $g$ sera donné par

$$
G(\xi)=\sum_{n}^{0} a_{k} \xi^{k}+\int_{\gamma} \varphi(x) e^{-x \xi} d x
$$

avec $\gamma$ le segment $\left[0, \varepsilon e^{i \theta}\right]$ de la demi-droite $\delta_{0, \theta}$; changer $\varepsilon$ ne modifie $G$ que par un terme à décroissance exponentielle. Il est facile de voir que $G$ admet la série $\hat{g}(\xi)=\sum_{n}^{-\infty} a_{k} \xi^{k}$ comme développement asymptotique, uniformément dans tout secteur $|\tau+\theta| \leq \frac{\pi}{2}-\delta$, $\delta>0$ (voir un peu plus loin au $\S 7$ des calculs plus précis). Cela nous amène aux définitions et aux remarques suivantes.

(5.3.1). Soit $\mathcal{A} \subset \mathcal{A}^{<1}$ le faisceau sur $S$ ainsi défini: $\mathcal{A}_{\tau}$ est formé des $g$ holomorphes dans un $U \in \mathcal{V}(\tau)$ et admettant dans $U$, pour $\xi \rightarrow \infty$, un développement asymptotique à tous les ordres appartenant à $\hat{K}=\mathbf{C}\left[\left[\frac{1}{\xi}\right]\right][\xi]$.

(5.3.2). Soit, pour $s \geq 0, K_{s}$ le sous-ensemble (en fait, le souscorps) de $\hat{K}$ formé des séries $\sum_{k \leq m} a_{k} \xi^{k}$ telles que la série $\sum_{\ell \geq 0} \frac{a_{-\ell}}{(\ell !)^{\bullet}} t^{\ell}$ converge; alors l'application composée

$$
\mathcal{C}_{o} \stackrel{\mathcal{F}}{\longrightarrow} \Gamma\left(S, \mathcal{A} / \mathcal{A}^{\leq-1}\right) \text { développ. } \stackrel{\text { asymptotique }}{\longrightarrow} \hat{K}
$$




\section{Équations Différentielles Linéaires}

est un isomorphisme $\mathcal{C}_{o} \stackrel{\sim}{\longrightarrow} K_{1}$.

En fait, on peut démontrer aussi que $\Gamma\left(S, \mathcal{A} / \mathcal{A}^{\leq-1}\right)$ est isomorphe à ces deux espaces; ceci peut se faire (au moins) de deux manières; la première consisterait à démontrer directement en utilisant la transformation de Fourier inverse l'isomorphisme $\mathcal{F}: \mathcal{C}_{o} \stackrel{\sim}{\longrightarrow} \Gamma\left(S, \mathcal{A} / \mathcal{A}^{\leq-1}\right)$; je la laisse au lecteur à titre d'exercice. La seconde consiste à démontrer directement l'isomorphisme avec $K_{1}$; elle sera donnée au $\S 7$.

\section{$\S 6$ Sommabilité de Borel}

Je noterai $\hat{\mathcal{F}}$ l'isomorphisme (5.3.2): $\mathcal{C}_{o} \stackrel{\sim}{\longrightarrow} K_{1}$, et $\mathcal{B}$ l'isomophisme inverse; au langage près (microfonction, " $\delta$ ", etc...), ils sont bien classiques; $\mathcal{B}$ est la transformation de Borel, qui sert de point de départ à sa théorie des séries divergentes [Bo]. Je rappelle rapidement quelques points essentiels de cette théorie.

DÉfinition (6.1). Soit $\hat{g} \in K_{1}$. On dit que $\hat{g}$ est sommable-Borel sur $I_{\theta}$ si sa transformée de Borel $f=\mathcal{B} \hat{g}$ a la propriété suivante: var $f$ se prolonge en une fonction holomorphe à croissance exponentielle à l'infini dans un secteur $|\arg x-\theta|<\varepsilon$. Si l'on note $F$ l'hyperfonction ainsi définie, sa transformée de Fourier $g$ (qui appartient à $\Gamma\left(I_{\theta}, \mathcal{A}\right)$, et admet $\hat{g}$ pour développement asymptotique à l'infini) s'appelle "la somme de Borel" de $\hat{g}$ sur $I_{\theta}$.

La fonction $g$ est, dans le secteur considéré, une "somme" de $\hat{g}$ qui en un sens, est meilleure que toutes les autres. Bien entendu, si 


\section{B. Malgrange}

$\hat{g}$ est déjà convergente, elle est sommable sur tout $I_{\theta}$ et sa somme lui est égale (démonstration laissée au lecteur).

On peut caractériser directement les fonctions qui sont sommes de Borel. Pour cela, introduisons la définition suivante:

DÉfinition (6.2). Pour $s \geq 0$ on désigne par $\mathcal{A}_{(s)}$ le sous-faisceau sur $S$ de $\mathcal{A}$ défini ainsi: soit $g \in \mathcal{A}_{\tau}$, de développement asymptotique $\hat{g}=\sum_{k \geq-m} a_{k} / \xi^{k} ;$ alors $g \in \mathcal{A}_{(s), \tau}$ si, dans un secteur $U \in \mathcal{V}(\tau)$, il vérifie pour tout $n \geq 1$ les inégalités $\left|g(\xi)-\sum_{k \leq n-1} a_{k} / \xi^{k}\right| \leq$ $(n !)^{s}\left(\frac{C}{\xi}\right)^{n}$, avec $C>0$ indépendant de $n$.

Il est immédiat que si $g \in \mathcal{A}_{(s)}$, on a $\hat{g} \in K_{s}$ (mais la réciproque est évidemment fausse!). Avec la définition précédente, on a le théorème classique suivant

ThÉorÈme (6.3). (F. Nevanlinna). Soit I un intervalle fermé de $S$ de longueur $\pi$, et soit $g \in \Gamma(I, \mathcal{A})$, de développement asymptotique $\hat{g}$. Pour que $\hat{g}$ soit sommable-Borel sur $I$, de somme $g$, il faut et il suffit qu'on ait $g \in \Gamma\left(I, \mathcal{A}_{(1)}\right)$.

Une démonstration de ce théorème sera donnée au $§ 7$, comme conséquence d'une étude plus systématique du faisceau $\mathcal{A}_{(1)}$.

(6.4) Je signale aussi, sans entrer dans les détails, l'existence de procédés "effectifs" de sommation des séries sommables-Borel; ces procédés se fondent sur la méthode suivante: une transformation conforme convenable permet d'exprimer $\operatorname{var}(\mathcal{B} \hat{g})$ sur la demi-droite $\delta_{0, \theta}$ en fonction de son développement à l'origine; en intégrant terme à terme, on trouve une série convergente qui représente $g$, et dont chaque coefficient se calcule avec un nombre fini de termes de $\hat{g}$. Le 


\section{Équations Différentielles Linéaires}

procédé le plus classique est celui qui consiste à transformer la partie positive $\sum_{n \geq 0} \frac{a_{n}}{\xi^{n}}$ de $\hat{g}$ en une série de factorielles $\sum_{n \leq 0} \frac{b_{n}}{\xi(\xi+\omega) \ldots(\xi+n \omega)}$, avec $\omega=r e^{-i \theta}, r>>0$. (Il est clair que $b_{n}$ ne va dépendre que de $a_{o}, \ldots, a_{n+r}$; en fait les formules pour passer des " $a$ " aux " $b$ " et vice-versa constituent ce qu'on appele l'algorithme de Stirling; cf [W]). Sur ces questions, voir notamment [Bo], chap 6, et [W].

(6.5) L'application aux équations différentielles à coefficients polynomiaux est immédiate: soit $p\left(x, \partial_{x}\right)$ un opérateur différentiel vérifiant (1.5); supposons que $p$ ait une singularité régulière en 0 (ou en un autre point; on se ramène au cas précédent par une translation), soit $f \in \mathcal{C}$, solution de $p f=0 ; f$ est de classe de Nilsson, et, pour toutes les directions $\theta$ sauf un nombre fini, var $f$ se prolonge en une fonction holomorphe à croissance exponentielle dans un secteur $|\arg x-\theta|<\varepsilon$. Si donc on suppose en outre $f$ "régulière" (cette hypothèse étant d'ailleurs inessentielle, $\mathrm{cf}(5.2)$ ), on sera dans les conditions de la définition (6.1); la solution formelle $\hat{g}=\hat{\mathcal{F}} f$ de $q=\mathcal{F} p$ sera sommable-Borel sur $I_{\theta}$, et sa somme $g$ vérifiera $q g=0$.

J'examinerai plus loin, au $\S 8$, l'extension de ce résultat aux systèmes du type considéré en (4.3).

\section{$\S 7$ Interprétation cohomologique}

On doit à Ramis [Ra 1], [Ra 2] (voir aussi [M-R]) une version "cohomologique" de la sommabilité à la Borel; elle repose sur une extension aux classes de Gevrey des calculs de Sibuya et de l'auteur 


\section{B. Malgrange}

[Si], [Ma 2]; pour la commodité du lecteur, je rappellerai d'abord ces derniers.

(7.1) Les notations $\mathcal{A}, \hat{K}, K_{s}$, ont la même signification qu'en (5.3). On pose $K_{o}=K\left(=\right.$ le corps de fonctions méromorphes $\left.\mathbf{C}\left\{\frac{1}{\xi}\right\}[\xi]\right)$; on note $\mathcal{A}^{<0}$ le sous-faisceau de $\mathcal{A}$ des fonctions à décroissance modérée, i.e. plus rapide que toute puissance de $\frac{1}{\xi}$; il revient au même de dire que ce sont les éléments de $\mathcal{A}$ dont le développement asymptotique est nul.

Par ailleurs, il sera plus commode ici de se ramener à l'origine, par le changement de variables $t=\frac{1}{\xi}$.

La proposition suivante est bien classique.

Proposition (7.1.1). (Borel-Ritt). Soit $\hat{g} \in \hat{K}$, et soit I un interalle ouvert de $S$ (ou même de $\tilde{S}$, le revêtement universel de $S$ ). Il existe $g \in \Gamma(I, \mathcal{A})$ admettant $\hat{g}$ comme développement asymptotique.

Voici une démonstration rapide, copiée dans $[\mathbf{W}]$ : par une ramification, on se ramène au cas où $I$ est de longueur $\leq \pi$; on peut alors supposer $I=]-\frac{\pi}{2}, \frac{\pi}{2}$ [; quitte à supprimer un nombre fini de termes, on peut supposer $\hat{g}$ sans pôle, i.e. $\hat{g}=\sum_{n \leq 0} a_{n} t^{n}$; alors on pose $g=\sum^{\prime} a_{n} t^{n}\left(1-e^{-1 /\left|a_{n}\right| t}\right)$, le symbole $\sum^{\prime}$ signifiant qu'on somme sur les $n$ où $a_{n} \neq 0$. Le résultat se déduit facilement du fait suivant: pour $\operatorname{Re} t>0$, on a $\left|1-e^{-t}\right|=\left|\int_{[0, t]} e^{-s} d s\right| \leq|t|$.

Ce résultat nous donne en particulier un isomorphisme $\mathcal{A} / \mathcal{A}^{<0} \stackrel{\sim}{\longrightarrow} \hat{K}$ (le second membre est considéré comme faisceau constant sur $S$ ). On considère alors la suite exacte $0 \rightarrow \mathcal{A}^{<0} \rightarrow$ 


\section{Équations Différentielles Linéaires}

$\mathcal{A} \rightarrow \mathcal{A} / \mathcal{A}^{<0} \rightarrow 0$ et la suite exacte de cohomologie

$$
\begin{aligned}
0 \rightarrow H^{o}\left(S, \mathcal{A}^{<0}\right) & \rightarrow H^{o}(S, \mathcal{A}) \rightarrow H^{o}\left(S, \mathcal{A} / \mathcal{A}^{<0}\right) \rightarrow H^{1}\left(S, \mathcal{A}^{<0}\right) \\
& \rightarrow H^{1}(S, \mathcal{A}) \rightarrow H^{1}\left(S, \mathcal{A} / \mathcal{A}^{<0}\right) \rightarrow 0 .
\end{aligned}
$$

On a évidemment $H^{\circ}\left(S, \mathcal{A}^{<0}\right)=0$, et $H^{\circ}(S, \mathcal{A})=K$; par ailleurs la proposition précédente nous donne $H^{\circ}\left(S, \mathcal{A} / \mathcal{A}^{<0}\right)=\hat{K}$; d'où une flèche $\hat{K} / K \rightarrow H^{1}\left(S, \mathcal{A}^{<0}\right)$.

Proposition (7.1.2). Cette flèche est un isomorphisme.

Cette proposition résulte immédiatement du lemme suivant

Lemme (7.1.3). (Sibuya). La flèche $H^{1}\left(S, \mathcal{A}^{<0}\right) \rightarrow H^{1}(S, \mathcal{A})$ est nulle.

Soit $\left\{U_{i}\right\}$ un recouvrement ouvert de $S$; en prenant un raffinement, on peut supposer que les $U_{i}$ sont des intervalles, et que les intersections 3 à 3 sont vides. Soient alors $f_{i j} \in \Gamma\left(U_{i} \cap U_{j}, \mathcal{A}^{<0}\right)$ avec $f_{i j}=-\delta_{i j}$ il s'agit de trouver des $g_{i} \in \Gamma\left(U_{i}, \mathcal{A}\right)$ tels qu'on ait $f_{i j}=g_{i}-g_{j}$. Par linéarité, on peut supposer que tous les $f_{i j}$ sont nuls sauf pour $(i, j)=(1,2)$ ou $(2,1)$. Alors en posant $\left.f_{12}=f, U_{12}=U=\right] \alpha, \beta[$, le résultat à démontrer est le suivant: il existe $g$, section de $\mathcal{A}$ sur $] \alpha, \beta+2 \pi[$ tel que la différence des deux déterminations de $g$ soit égale à $f$.

Quite à rétrécir $U$, on peut supposer $f$ défini sur $|t|<2 r$, $\alpha<\arg t<\beta$; soit $\gamma \in] \alpha, \beta$ [; on prend $g(t)=\frac{-1}{2 \pi i} \int_{\delta} \frac{f(s)}{s-t} d s$ avec $\delta$ le segment $\left[0, r e^{i \gamma}\right]$; alors $g$ est défini et holomorphe dans $\mathbf{C}-\delta$; sur l'intérieur de $\delta$, la différence de ses deux déterminations vaut $f$; donc $g$ se prolonge en une fonction holomorphe dans $\{\alpha<\arg t<$ $\beta,|t|<r\}$, vérifiant $g\left(e^{2 \pi i} t\right)-g(t)=f(t)$.

Reste à établir l'existence d'un développement asymptotique en 0 ; il suffit d'établir le résultat dans un secteur $\gamma+\varepsilon<\arg x<$ 


\section{B. Malgrange}

$\gamma+2 \pi-\varepsilon$. (En effet, pour établir ensuite le résultat au voisinage de $\delta$, on pourra remplacer $\delta$ par un segment $\delta^{\prime}=\left[0, r e^{i \gamma^{\prime}}\right]$ et l'arc de cercle joignant $r e^{i \gamma} \grave{\text { à }} r e^{i \gamma^{\prime}}$ ).

Posons $a_{k}=-\frac{1}{2 \pi i} \int_{\delta} \frac{f(s)}{s^{k+\jmath}} d s$ et montrons que, dans le secteur considéré, $g$ admet $\sum_{k \leq 0} a_{k} t^{k}$ pour développement asymptotique; on a

$$
g(t)-\sum_{0}^{n} a_{k} t^{k}=-\frac{1}{2 \pi i} \int_{\delta} \frac{t^{n+1}}{s^{n+1}} \frac{f(s)}{s-t} d s
$$

et le résultat se déduit immédiatement de là, et du fait que le développement asymptotique de $f$ est nul. D'où le lemme.

Je renvoie aux articles cités et à [Ma 3] pour les applications de ces arguments (et de leur "variante nonlinéaire") aux équations différentielles; la principale application concerne la classification des équations, ou des systèmes, de forme normale formelle donnée. Une autre concerne "l'irrégularité": soit $p\left(t, \partial_{t}\right)$ une équation à coefficients dans $\mathcal{O}$ (ou $K$ ); le théorème des développements asymptotiques nous dit que $p$ : $\mathcal{A}^{<0} \rightarrow \mathcal{A}^{<0}$ est surjectif; notons $\mathcal{A}^{<0}(p)$ pour le noyau, qui est le faisceau des solutions de $p f=0$ à décroissance plus rapide que toute puissance de $t$; on trouve alors un isomorphisme $H^{1}\left(S, \mathcal{A}^{<0}(p)\right) \simeq \operatorname{ker}(p, \hat{K} / K)$; le second membre exprime justement "l'irrégularité" de $p$, i.e. la différence de comportement de $p$ quand on passe des séries formelles aux séries convergentes. Cf loc. cit.

(7.2) Suivant Ramis, loc. cit, nous allons maintenant reprendre les mêmes raisonnements, en ajoutant des conditions de Gevrey, c'està-dire nous allons travailler avec $\mathcal{A}_{(1)}$ au lieu de $\mathcal{A}$ (en fait, on peut aussi faire la même chose avec $s$ quelconque, mais peu importe ici). L'analogue de (7.1.1) est la proposition suivante 


\section{Équations Différentielles Linéaires}

Proposition (7.2.1). Soit I un intervalle ouvert de longueur $\leq \pi$, et soit $\hat{g} \in K_{1}$; alors il existe $g \in \Gamma\left(I, \mathcal{A}_{(1)}\right)$ admettant $\hat{g}$ pour développement asymptotique.

Il est plus naturel ici de repasser en $\xi=1 / t$; on peut supposer aussi qu'on ait $I=$ ] $-\frac{\pi}{2}, \frac{\pi}{2}$ [ et que $g$ n'ait pas de terme polaire ni de terme constant, i.e. s'écrive $g(\xi)=\sum_{k \geq 1} a_{k} / \xi^{k}$. Soit $\varphi Y$ son transformé de Borel, avec $\varphi(x)=\sum_{k \geq 0} \frac{a_{k+1}}{k !} x^{k}$. Soit $R$ le rayon de convergence de $\varphi$; quitte à faire une homothétie, on peut supposer $R>1$. On prend alors

$$
g(\xi)=\int_{0}^{1} \varphi(x) e^{-x \xi} d x
$$

on va voir que $g$ répond à la question; soit $\delta>0$; on va faire les majorations dans le secteur $|\arg \xi| \leq \frac{x}{2}-\delta$; dans ce secteur, on a $\left|e^{-x \xi}\right| \leq e^{-x \rho}$, avec $\rho=|\xi| \sin \delta$. On a

$$
\begin{aligned}
& g(\xi)-\sum_{0}^{n} a_{k} / \xi^{k}=\int_{0}^{1} \varphi(x) e^{-x \xi} d x-\sum_{0}^{n-1} \frac{a_{k+1}}{k !} \int_{0}^{\infty} x^{k} e^{-x \xi} d x \\
& =\sum_{n}^{\infty} \frac{a_{k+1}}{k !} \int_{0}^{1} x^{k} e^{-x / x i} d x-\sum_{0}^{n-1} \frac{a_{k+1}}{k !} \int_{1}^{\infty} x^{k} e^{-x \xi} d x .
\end{aligned}
$$

On majore les premiers (resp. les derniers termes) en remplaçant $x^{k}$ par $x^{n}$, ce qui marche dans les deux cas (!), $e^{-x \xi}$ par $e^{-x \rho}$, et enfin $\int_{0}^{1}$ et $\int_{1}^{\infty} \operatorname{par} \int_{0}^{\infty} ;$ finalement, on trouve

$$
\left|g(x)-\sum_{1}^{n} a_{k} / \xi^{k}\right| \leq C \int_{0}^{\infty} x^{n} e^{-x \rho} d x=\frac{C n !}{\rho^{n+1}}
$$




\section{B. Malgrange}

avec $C=\sum \frac{\left|a_{k+1}\right|}{k !}$ (qui est fini puisque le rayon de convergence de $\varphi$ est $>1)$. D'où le résultat.

Ceci nous donne en particulier un isomorphisme $\mathcal{A}_{(1)} / \mathcal{A}_{(1)} \cap$ $\mathcal{A}^{<0} \simeq K_{1}$.

Lemme (7.2.2). On a $\mathcal{A}_{(1)} \cap \mathcal{A}^{<0}=\mathcal{A} \leq-1$, le faisceau des fonctions à décroissance exponentielle.

Soit $g$ holomorphe dans un secteur $U$ et y vérifiant, pour tout $n \geq 1,|g(\xi)| \leq n !\left(\frac{A}{\xi}\right)^{n}$; on choisit $n=$ (partie entière de $\left.\frac{\xi}{A}\right)$; en appliquant Stirling, on voit que $g$ est à décroissance exponentielle.

Réciproquement, si $g$ vérifie, dans $U:|g(\xi)| \leq C e^{-A|\xi|}$, on a $\left|\xi^{n} g(\xi)\right| \leq C \exp (-A|\xi|+n \log |\xi|)$; le maximum de second membre est obtenu pour $|\xi|=\frac{n}{A}$, et on conclut par Stirling.

Remarque: Prenons plus généralement $g$ une section de $\mathcal{A}_{(1)}$; alors, dans un secteur $U$ on aura $\left|g(\xi)-\sum_{k \leq n-1} a_{k} / \xi^{k}\right| \leq n !\left|\frac{A}{\xi}\right|^{n} ;$ si l'on choisit alors $n=$ (partie entière de $\frac{\xi}{A}$ ), comme au lemme précédent, on aura une approximation exponentiellement petite de $g$. Réciproquement, ceci caractérise $\mathcal{A}_{(1)}$, en raisonnant comme dans la réciproque ci-dessous.

D'une manière intuitive (et légèrement incorrecte), ceci veut dire qu'on obtient une bonne approximation, en fait exponetiellement petite, en faisant ce que Poincaré appelait la "sommation au sens des astronomes", qui consiste à couper une série asymptotique au plus petit terme. En particulier, ce que nous faisons ici permet de donner une justification à ce type de procédures, lorsqu'on les applique à des solutions d'équations différentielles.

Le lemme suivant est la version Gevrey de (7.1.3). 


\section{Équations Différentielles Linéaires}

Lemme (7.2.3). La flèche $H^{1}\left(S, \mathcal{A}^{\leq-1}\right) \rightarrow H^{1}\left(S, \mathcal{A}_{(1)}\right)$ est nulle.

Il suffit de reprendre la démonstration de (7.2.3) en vérifiant que, si $f$ est à décroissance exponentielle, $g$ vérifie la condition de Gevrey. Ceci résulte du même type de majorations qu'au lemme précédent.

Nous pouvons maintenant repéter les arguments de (7.1); par (7.2.1) et (7.2.2) on a $\mathcal{A}_{(1)} / \mathcal{A}^{\leq-1} \simeq K_{1}$; en utilisant la suite exacte de cohomologie et (7.2.4) on trouve finalement le résultat suivant ThÉorème (7.2.5). On a des isomorphismes $H^{\circ}\left(S, \mathcal{A}_{(1)} / \mathcal{A}^{\leq-1}\right) \simeq$ $K_{1}$, et $H^{1}\left(S, \mathcal{A}^{\leq-1}\right) \simeq K_{1} / K$.

Montrons aussi le résultat laissé en suspens en (5.3.2).

Proposition (7.2.6). L'injection naturelle $\mathcal{A}_{(1)} \rightarrow \mathcal{A}$ donne un isomorphisme $\left(K_{1}=\right) H^{o}\left(S, \mathcal{A}_{(1)} / \mathcal{A}^{\leq-1}\right) \stackrel{\sim}{\sim} H^{o}\left(S, \mathcal{A} / \mathcal{A}^{\leq-1}\right)$.

En effet, on considère les deux suites exactes

$$
\begin{aligned}
& 0 \rightarrow H^{o}\left(S, \mathcal{A}_{(1)}\right) \rightarrow H^{o}\left(S, \mathcal{A}_{(1)} / \mathcal{A}^{\leq-1}\right) \rightarrow H^{1}\left(S, \mathcal{A}^{\leq-1}\right) \rightarrow 0 \\
& 0 \rightarrow H^{o}(S, \mathcal{A}) \rightarrow H^{o}\left(S, \mathcal{A} / \mathcal{A}^{\leq-1}\right) \quad \rightarrow H^{1}\left(S, \mathcal{A}^{\leq-1}\right) \rightarrow 0
\end{aligned}
$$

La première flèche verticale est un isomorphisme (les deux termes valent $K$ ); la dernière l'est aussi. Donc il en va de même de la seconde.

(7.3)Applications à la sommabilité de Borel

Notons d'abord les résultats suivants

(7.3.1j. Si $I$ est un intervalle fermé de $S$ de longueur $\pi$, on a $H^{o}\left(I, \mathcal{A}^{\leq-1}\right)=0$. 


\section{B. Malgrange}

En effet, soit $g \in H^{o}\left(I, \mathcal{A}^{\leq-1}\right)$; les raisonnements de (4.1) montrent que sa transformée de Fourier inverse est nulle; donc $g=0$. Une autre démonstration, plus classique, consiste à appliquer la méthode de Phragmén-Lindelöf.

Le même résultat reste vrai si $I$ est semi-fermé de longueur $\pi$; par contre, il devient fausse si $I$ est ouvert de longueur $\pi$, ou contenu dans un tel intervalle (prendre $f=e^{\alpha \xi}, \alpha$ convenable).

(7.3.2). Si $I$ est un intervalle fermé de $S$ de longueur $<\pi$, on a $H^{1}\left(I, \mathcal{A}^{\leq-1}\right)=0$.

On considère en effet la suite exacte $0 \rightarrow \mathcal{A}^{\leq-1} \rightarrow \mathcal{A}_{(1)} \rightarrow$ $\mathcal{A}_{(1)} / \mathcal{A}^{\leq-1} \rightarrow 0$. On prend sa suite exacte de cohomologie sur $I$

$$
\begin{aligned}
0 & \rightarrow H^{o}\left(I, \mathcal{A}^{\leq-1}\right) \rightarrow H^{o}\left(I, \mathcal{A}_{(1)}\right) \rightarrow H^{o}\left(I, \mathcal{A}_{(1)} / \mathcal{A}^{\leq-1}\right) \rightarrow \\
& \rightarrow H^{1}\left(I, \mathcal{A}^{\leq-1}\right) \rightarrow H^{1}\left(I, \mathcal{A}_{(1)}\right)
\end{aligned}
$$

La dernière flèche est nulle par le même raisonnement qu'en (7.2.4); d'autre part (7.2.1) nous dit que la flèche $H^{\circ}\left(I, \mathcal{A}_{(1)}\right) \rightarrow K_{1}$ est surjective. Donc $H^{1}\left(I, \mathcal{A}^{\leq-1}\right)=0$.

Il me parait probable que le résultat subsiste pour $I$ ouvert de longueur $\leq \pi$ (il y a un passage à la limite que je ne vois pas). Par contre les résultats que nos allons établir montrent qu'il n'en est pas de même pour $I$ fermé de longueur $\geq \pi$; on peut voir qu'il n'en est pas non plus de même pour $I$ semi-fermé de longueur $\geq \pi$.

(7.3.3) Démontrons maintenant le Théorème (6.3). Prenons par exemple $I=\left[-\frac{\pi}{2}, \frac{\pi}{2}\right]$, et soit $\hat{g} \in K_{1}$, sommable-Borel sur $I$; soit $f=\mathcal{B} \hat{g} \in \mathcal{C}_{o}$ sa transformée de Borel; on peut supposer $\hat{g}$ sans partie polaire donc $f=\varphi Y$, avec $\varphi$ holomorphe en 0 , se prolongeant en 


\section{Équations Différentielles Linéaires}

une fonction à croisance exponentielle dans un secteur $|\arg x|<\varepsilon$; on a alors

$$
g(\xi)=\int_{\delta_{0, \theta}} \varphi(x) e^{-x \xi} d x, \quad(|\theta|<\varepsilon)
$$

Il suffit de faire les majorations dans $|\arg \xi| \leq \frac{\pi}{2}-\eta, \eta>0$ quelconque, en intégrant le long de $\mathbf{R}_{+}$(on les obtiendra ailleurs en changeant le chemin d'intégration). Supposons p.ex. que le rayon de convergence de $\varphi$ soit $>1$. On écrit alors

$$
g(\xi)=\int_{0}^{1} \varphi(\xi) e^{x \xi} d x+\int_{1}^{\infty} \varphi(\xi) e^{-x \xi} d x
$$

Le premier terme se majore comme dans (7.2.1). Le second est à décroissance exponentielle, donc la majoration résulte ici de (7.2.2); dans les deux cas, on trouve une section de $\mathcal{A}_{(1)}$; d'où le résultat.

Démontrons maintenant la réciproque; la méthode que je vais employer n'est évidemment pas tout à fait la méthode classique; mais elle a l'avantage d'être naturelle dans le contexte considéré ici. Soit $g \in \Gamma\left(I, \mathcal{A}_{(1)}\right)$, avec $I=\left[-\frac{\pi}{2}, \frac{\pi}{2}\right]$; la transformée de Fourier inverse de $g$ a les propriétés de croissance voulues à l'infini; si l'on note par $f$ la microfonction qu'elle définit en 0 , tout revient à montrer qu'on a $f \in \mathcal{C}_{o}$; or $f$ ne dépend que de la classe définie par $g$ dans $\Gamma\left(I, \mathcal{A}^{<1} / \mathcal{A}^{\leq-1}\right)$, a fortiori de la classe qu'il définit dans $\Gamma\left(I, \mathcal{A}_{(1)} / \mathcal{A}^{\leq-1}\right)$; or cette classe est $\hat{g} \in K_{1}$ le développement asymptotique de $g$, et l'on aura donc $f=\mathcal{B} \hat{g}$. D'où le résultat.

Voici le résultat final de ce paragraphe.

ThÉORÈme (7.3.4). Soit $\hat{g} \in K_{1}$, et soient $\alpha(\hat{g}), \beta(\hat{g}), \gamma(\hat{g})$ respectivement les classes qu'il définit dans $H^{o}\left(S, \mathcal{A}_{(1)} / \mathcal{A}^{\leq-1}\right)$, dans 


\section{B. Malgrange}

$H^{o}\left(S, \mathcal{A} / \mathcal{A}^{\leq-1}\right)$, et dans $H^{1}\left(S, \mathcal{A}^{\leq-1}\right)$. Soit $I$ un intervalle fermé de $S$, de longueur $\pi$. Les propriétés suivantes sont équivalentes

i) $\hat{g}$ est sommable-Borel sur $I$

ii) $\alpha(\hat{g}) \mid I$ se relève en une section de $H^{o}\left(I, \mathcal{A}_{(1)}\right)$

iii) $\beta(\hat{g}) \mid I$ se relève en une section de $H^{o}(I, \mathcal{A})$

iv) $\gamma(\hat{g}) \mid I=0$.

Le Théorème (6.3) montre l'équivalence i) $\Leftrightarrow$ ii). L'équivalence ii) $\Leftrightarrow$ iv) résulte de ce que, dans la suite exacte

$$
H^{o}\left(I, \mathcal{A}_{(1)}\right) \rightarrow H^{o}\left(I, \mathcal{A}_{(1)} / \mathcal{A}^{\leq-1}\right) \rightarrow H^{1}\left(I, \mathcal{A}^{\leq-1}\right) \rightarrow H^{1}\left(I, \mathcal{A}_{(1)}\right),
$$

la dernière flèche est nulle par (7.2.4). L'équivalence iii) $\Leftrightarrow$ iv) se démontre de la même manière. D'où le théorème.

Remarque (7.3.5): Bien entendu, si $\hat{g}$ est sommable-Borel sur $I$, le relèvement sur $I$ de $\alpha(\hat{g})$ et celui de $\beta(\hat{g})$ sont uniques, et égaux à la somme de $\hat{g}$ sur $I$.

\section{$\S 8$ Application aux équations différentielles}

Je n'en dirai que quelques mots; je vais examiner rapidement l'extension de (6.5) aux systèmes du type considéré en (4.3).

Considérons un système $\partial_{\xi} G+M G=0, M$ une matrice carrée $n \times n$ holomorphe à l'infini: $M=M_{o}+\frac{M_{1}}{\xi}+\ldots$; soient $\left(\alpha_{1}, \ldots, \alpha_{p}\right)$ les valeurs propres de $M_{o}$; je suppose p.ex. $\alpha_{1}=0$ (si non, on s'y ramène en faisant le changement $G^{\prime}=e^{\alpha_{1} \xi} G$ ). On sait alors (lemme de Sibuya; cf $[\mathbf{L}]$ ou $[\mathbf{W}])$, qu'il existe un changement de base formel 


\section{Équations Différentielles Linéaires}

$G=S H, S \in G \ell(n, \hat{K})$ tel que, dans la nouvelle base, le système se décompose en systèmes

$$
\partial_{\xi} H^{(k)}+M^{(k)} H^{(k)}=0, \quad \text { avec } \quad M^{(k)}=M_{o}^{(k)}+\frac{M_{1}^{(k)}}{\xi}+\ldots,
$$

$M_{o}^{(k)}$ ayant $\alpha_{k}$ pour unique valeur propre.

L'hypothèse qui généralise celle faite en (6.5) est la suivante (j'admets ce point):

(8.2). $M^{(1)}$ est à singularité régulière.

Soit $\hat{H}^{(1)}$ une solution formelle de (8.1.1); pour rester dans le cadre considéré ici, je suppose que ces coefficients appartiennent à $\hat{K}$ (mais il est entendu que tout marcherait de la même manière s'ils étaient "de Nilsson formels"; $\mathrm{cf}(5.2))$. En revenant à l'équation initiale, on trouve une solution formelle $\hat{G} \in \hat{K}^{n}$ de $\partial_{\xi} G+M G=0$. On peut établir les propriétés suivantes (cf [Ma 3], par exemple)

i) Sur tout intervalle fermé $I$ de longueur $\pi$, dont les extrémités ne sont pas des lignes de Stokes, $\hat{G}$ se relève en une solution $G_{1} \in \Gamma(I, \mathcal{A})$.

ii) Sur l'intersection de deux tels intervalles, disons $I$ et $\mathcal{J}$, $G_{I}-G_{\mathcal{J}}$ est à décroissance exponentielle (c'est ici que (8.2) intervient).

Ces propriétés nous disent d'abord que $\hat{G}$ est le développement asymptotique d'une section sur $S$ de $\left(\mathcal{A} / \mathcal{A}^{\leq-1}\right)^{n}$; par conséquent, d'après (7.2.6), on a $\hat{G} \in K_{1}^{n}$. Alors (7.3.4) et (7.3.5) nous disent que sur tout $I$ vérifiant les conditions de i), $\hat{G}$ est sommable-Borel de somme $G_{I}$.

Pour d'autres applications, de la sommabilité de Borel, voir notamment [M-R]. 


\section{B. Malgrange}

N.B. Les trois exposés ci-dessus rédigés faisaient suite à deux autres, consacrés à la classification des équations à points singuliers irréguliers d'après Deligne, telle qu'elle est exposée dans [Ma 3]. Je me suis efforcé d'en rendre ces notes le plus indépendantes possible; cependant pour une compréhension plus complète des $\S(4.3)$ et 8 , la lecture de [Ma 3] est recommandée. 


\section{Équations Différentielles Linéaires}

\section{BIBLIOGRAPHIE}

[B] R.P. Boas, Entire functions, Academic Press (1954).

[B-K] L. Boutet de Monvel, P. Krée, Pseudo-differential operators and Gevrey classes, Ann. Inst. Fourier 17 (1967), 295-323.

[Bo] E. Borel, Leçons sur les séries divergentes, 2e. édition, Gauthier-Villars, Paris 1928.

[E] J. Ecalle, Les fonctions résurgentes, tomes I à III, Publications mathématiques d'Orsay (1981-85).

[I] E.L. Ince, Ordinary differential equations, Dover, New-York (1956).

[K] M. Kashiwara, Systems of microdifferential equations, Progress in Math., Birkhäuser (1983).

[L] A.H.M. Levelt, Jordan decomposition of a class of singular differential operators, Ark. Mat. 13 (1975), 1-27.

[Ma 1] B. Malgrange, Sur les points singuliers des équations différentielles, L'Enseignement Mathématiques 20, fasc. 1-2, (1974) 147-176.

[Ma 2] B. Malgrange, Remarques sur les équations différentielles à points singuliers irréguliers, Lect. Notes in Math. 712 (1979), 77-86, Springer-Verlag.

[Ma 3] B. Malgrange, La classification des connexions irrégulières à une variable, in Seminaires E.N.S. 1979-82, Progress in Math., Birkhäuser (1983).

[Ma 4] B. Malgrange, Introduction aux travaux de J. Ecalle, L'Enseignement Mathématique 31 (1985), 261-282.

[Ma 5] B. Malgrange, Transformation de Fourier géométrique, Séminaire Bourbaki no 692, février 1988.

[Ma 6] B. Malgrange, Systèmes holonomes à une variable (livre en préparation). 


\section{B. Malgrange}

[M-R] J. Martinet, J.P. Ramis, Problèmes de modules pour des équations différentielles non-linéaires, Publ. Math. I.H.E.S. no 55 (1982), 63-164.

[Ra 1] J.P. Ramis, Dévissage Gevrey, Astérisque 59-60 (1978), 173204.

[Ra 2] J.P. Ramis, Les séries $k$-sommables et leurs applications, Lect. Notes in Physics 126 (1980). Springer-Verlag.

[Ro] P. Robba, Lemme de Hensel pour des opérateurs différentiels, L'Enseignement Mathématique 26, fasc. 3-4 (1980), 279-311.

[S.K.K.] M. Sato, T. Kawai, M. Kashiwara, Hyperfunctions and pseudodifferential equations, Lect. Notes in Math. 287 (1973), 265-529, Springer-Verlag.

[Si] Y. Sibuya, Stokes phenomena, Bull. Amer. Math. Soc. 83 (1977), 1075-1077.

[W] W. Wasow, Asymptotic expansions for ordinary differential equations, Interscience Publ. (1965). 